Y. C. Minh and E. F. van Dishoeck, eds.

\title{
The Atmospheric Composition of the Giant Planets: Recent Discoveries from ISO and Galileo
}

\author{
Emmanuel Lellouch \\ Observatoire de Meudon, F-92195 Meudon, France
}

\begin{abstract}
The recent, concommitant, operation of the Galileo mission to the Jovian system and of the Infrared Space Observatory has provided a wealth of new results on the atmospheric composition of the Giant Planets. These results include (i) first or improved measurements of several elemental and isotope ratios, particularly in Jupiter; (ii) new insights into Jupiter's (and Saturn's) condensible species and tropospheric cloud structure; (iii) a much enriched view of stratospheric hydrocarbon chemistry; and (iv) the discovery of an external source of oxygen into the Giant Planets. These discoveries address key issues such as the origin of Giant Planets, their tropospheric meteorology and stratospheric physico-chemistry and dynamics, and their interaction with the interplanetary medium.
\end{abstract}

\section{Introduction}

There are classically two general reasons to study the composition of planetary atmospheres. The first one is to reconstruct the planetary history. The knowledge of molecular and atomic global abundances provides information on the elemental and isotopic ratios, which in turn constrain models of formation and evolution of the Giant Planets and of the Solar System as a whole. The other general reason is broadly speaking to understand the physics, chemistry and meteorology of current planetary atmospheres. Knowing the gas abundances, with their vertical and spatial variations, and the distribution of clouds, gives hints at a number of physico-chemical phenomena taking place in these atmospheres. These phenomena include condensation, chemistry and photochemistry, vertical and lateral transport and interaction with the planetary environments. The challenge is then to assemble all these constraints to build what can be called "meteorological" models, and it is particularly interesting to do so in a context of comparative planetology.

The present paper illustrates these aspects by presenting some of the most significant discoveries obtained by ISO and Galileo on the atmospheric composition of the Giant Planets. For completeness, a brief report on recent advances on the subject of elemental and isotope ratios is first given, sending the reader to the paper by Owen et al. (this volume) for much more details and an in-depth interpretation. The cloud structure and deep atmosphere meteorology of the Giant Planets is then discussed, with emphasis on Jupiter, as revealed primarily by Galileo. Recent progress of the hydrocarbon stratospheric chemistry and dynamics is then addressed. Finally, the problem of the external supply of oxygen 
to the Giant Planets is discussed. This gives an example of the emergence of a new (essentially introduced by ISO observations) subject linking planets with comets and the interplanetary environment.

A side aspect of this paper is to illustrate the synergy of interplanetary space missions and remote sensing. Indeed, it is sometimes (and not always without afterthoughts ... ) wondered what planetologists can still do from distant observations at the age of mature space exploration. Besides the need for monitoring time- and space-variable phenomena, which obviously cannot be performed by an in situ mission, it must be realized that telescopes on the ground or in Earth orbit have often more powerful instrumentation than contemporary interplanetary spacecrafts. Indeed, it is not possible for a 10-year long mission like Galileo to Jupiter or Cassini (currently en route to Saturn) to carry He-cooled instruments as ISO did. Therefore, although the infrared spectrum of planets had been largely explored by spacecraft observations (notably the IRIS observations on Voyager 1 and 2 for the Giant Planets and Titan), ISO turned out to be extremely competitive and achieved major discoveries in the field of planetary science (see review in Lellouch 1999). The major strength of ISO came from the enhanced spectral resolution of its spectrometers and the high sensitivity of all the instruments. This is particularly true for the ISO Short Wavelength Spectrometer (SWS) whose sensitivity and dynamical response appeared very well suited to the study of objects with flux levels ranging from less than $1 \mathrm{Jy}$ to $10^{6} \mathrm{Jy}$. The comparison between Saturn's spectrum at $11-16 \mu \mathrm{m}$ observed by Voyager 1-IRIS and by ISO-SWS (Fig. 1) demonstrates the importance of such improvements.

\section{Elemental and Isotopic Abundances in the Giant Planets}

\subsection{Elemental abundances in Jupiter: Galileo measurements}

Galileo obtained the first in situ determination of many elemental abundances in Jupiter's atmosphere from the mass spectrometer on its descent probe (Niemann et al. 1996,1998; Atreya et al. 1999). These measurements are gathered in Table 1. They indicate that none of the elemental abundances, when scaled to hydrogen, has a solar value. ${ }^{4} \mathrm{He}$ and ${ }^{20} \mathrm{Ne}$ are depleted in the observable atmosphere, an evidence for vertical segregation, with droplets of helium and neon falling towards the center of the planet. Other measured elements (C, $\mathrm{N}, \mathrm{S}, \mathrm{Ar}, \mathrm{Kr}, \mathrm{Xe}$ ) appear enriched compared to the Sun. This is evidence for a formation from icy planetesimals enriched in volatiles. Oxygen $(\mathrm{O} / \mathrm{H}=0.35$ times the solar value at $p=19$ bar in Jupiter's atmosphere) is at face value an exception, which can be explained by local meteorological effects, as discussed below. In the case of ${ }^{4} \mathrm{He}$ and $\mathrm{C}$, previously measured from Voyager-IRIS spectra 20 years ago, the in situ determination is a reassuring validation of remote spectroscopic sensing.

\subsection{Deuterium and other isotopic ratios in the Giant Planets}

The abundance of deuterium in Giant planets and comets is one of the keys to the understanding of their formation. Solar System objects formed from a protosolar nebula composed of gas and solid grains. In Jupiter and Saturn, the 

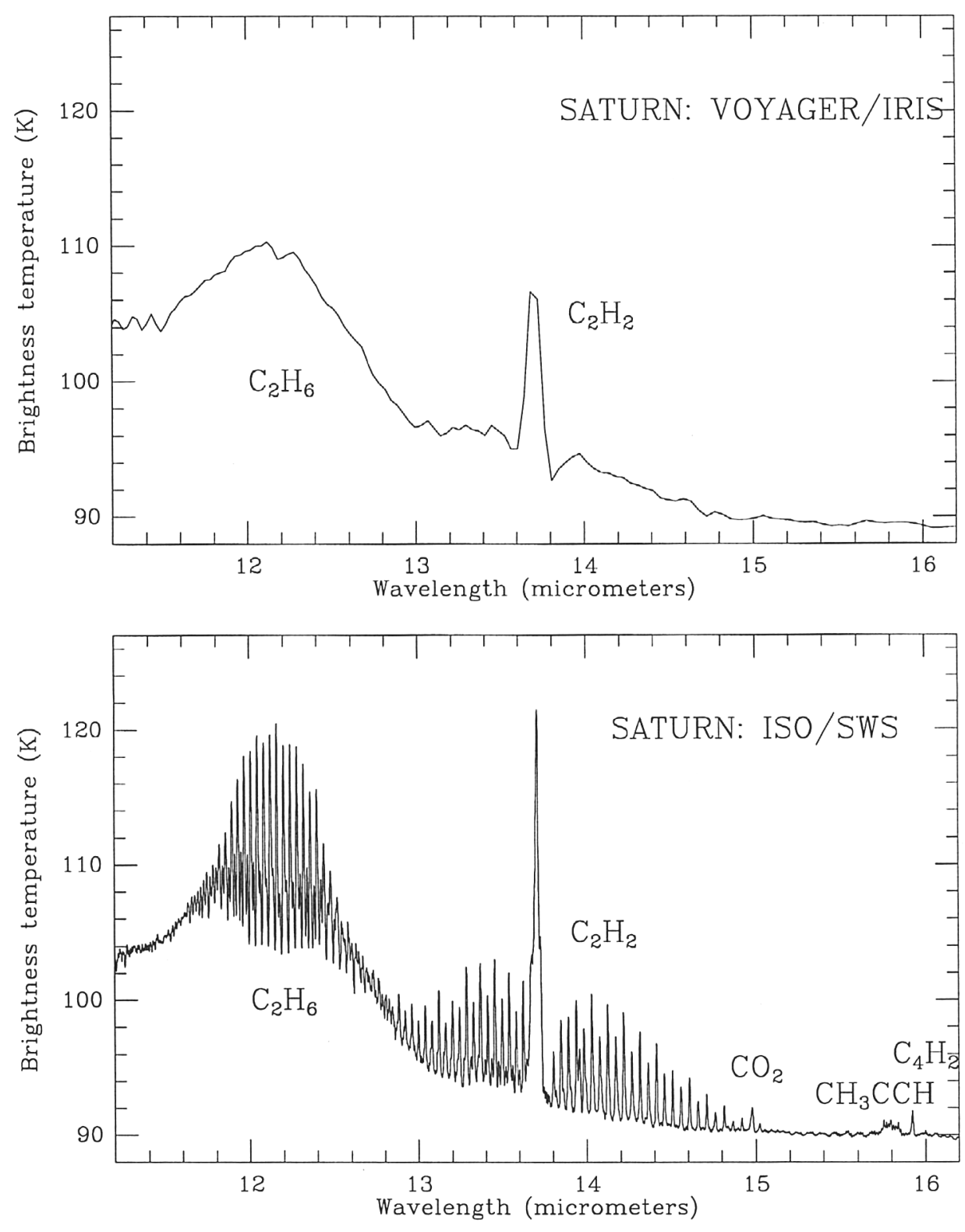

Figure 1. A comparison between Saturn's spectrum at 11-16 $\mu \mathrm{m}$, observed by Voyager 1-IRIS and by ISO-SWS. Note how ISO allows the resolution of the acetylene and ethane bands in full detail and the first detection of four new compounds: propyne at $15.8 \mu \mathrm{m}$, diacetylene at $15.9 \mu \mathrm{m}$, carbon dioxide at $14.98 \mu \mathrm{m}$ and benzene at $14.84 \mu \mathrm{m}$ (practically invisible on the figure). Adapted from de Graauw et al. (1997). 
Table 1. Elemental ratios in Jupiter.

\begin{tabular}{ccl}
\hline & Jupiter/Sun & Comment \\
\hline${ }^{4} \mathrm{He} / \mathrm{H}$ & $0.807 \pm 0.02$ & Galileo Probe \\
& $0.7 \pm 0.1$ & Voyager spectroscopy \\
${ }^{20} \mathrm{Ne} / \mathrm{H}$ & $0.10 \pm 0.01$ & Galileo Probe \\
${ }^{36} \mathrm{Ar} / \mathrm{H}$ & $1.7 \pm 0.6$ & $" \prime$ \\
${ }^{84} \mathrm{Kr} / \mathrm{H}$ & $3.0 \pm 1.0$ & $"$ \\
${ }^{132} \mathrm{Xe} / \mathrm{H}$ & $2.5 \pm 0.7$ & $"$ \\
$\mathrm{C} / \mathrm{H}$ & $2.9 \pm 0.5$ & \\
& $2.32 \pm 0.18$ & Voyager spectroscopy \\
$\mathrm{N} / \mathrm{H}$ & $3.6 \pm 0.5$ & Galileo Probe \\
$\mathrm{O} / \mathrm{H}$ & 0.35 & see text, Galileo Probe \\
\hline
\end{tabular}

vast majority of the mass in is fluid (as opposed to ice/rock) form; it is therefore regarded that the deuterium abundance in Jupiter and Saturn represents that of the gaseous part of the protosolar nebula. In contrast, Uranus and Neptune may have been enriched in deuterium by the mixing of their atmospheres with comparatively much larger cores (more than $50 \%$ of the mass) containing Drich icy grains. Therefore, comparing the $\mathrm{D} / \mathrm{H}$ ratio in Giant planets and using interior models may allow an estimate of the $\mathrm{D} / \mathrm{H}$ ratio in the protoplanetary grains that formed the cores of Uranus and Neptune.

This picture is supported by the available $\mathrm{D} / \mathrm{H}$ measurements in the Giant Planets and the comparison with the "protosolar nebula" value deduced from a model of the solar wind. However, with the exception of difficult and uncertain measurements at Jupiter and Saturn from the visible lines of HD, all determinations of $\mathrm{D} / \mathrm{H}$ in Giant Planets prior to ISO and Galileo relied on the atmospheric abundance of $\mathrm{CH}_{3} \mathrm{D}$. This is not a straightforward means to evaluate $\mathrm{D} / \mathrm{H}$ because of the existence of an isotopic exchange reaction between $\mathrm{CH}_{4}$ and $\mathrm{H}_{2}\left(\mathrm{CH}_{4}+\mathrm{HD} \rightleftarrows \mathrm{CH}_{3} \mathrm{D}+\mathrm{H}_{2}\right)$ whose equilibrium constant and kinetics are not well known:

$$
\mathrm{D} / \mathrm{H}=(1 / 4 f) \mathrm{CH}_{3} \mathrm{D} / \mathrm{CH}_{4}
$$

where $f$ is uncertain. The interest of Galileo is that it has provided the first in situ measurement of $\mathrm{D} / \mathrm{H}$ in a Giant Planet (Mahaffy et al. 1998). The interest of ISO is that it has allowed the first detection of the IR lines of HD, providing a coherent and direct determination of the bulk atmospheric $\mathrm{D} / \mathrm{H}\left(=1 /{ }_{2} \mathrm{HD} / \mathrm{H}_{2}\right)$ ratio in the four Giant planets. The D/H values from ISO (cf. Lellouch 1999 and references therein) generally compare well with earlier measurements, and, in Jupiter's case with the new Galileo value $\left((2.6 \pm 0.7) \times 10^{-5}\right)$. Although they are not yet very accurate, these measurements coherently confirm the picture of deuterium enrichment in Uranus and Neptune. The inferred $\mathrm{D} / \mathrm{H}$ ratio in the protouranian and protoneptunian ices, assuming full mixing of the atmosphere and central core, is however modest, $(10 \pm 5) \times 10^{-5}$. Thus these protoplanetary ices are significantly less D-rich than cometary ices (where $\mathrm{D} / \mathrm{H}$ is about $31 \times 10^{-5}$ ). This bears important implications on the relative origin of comets 

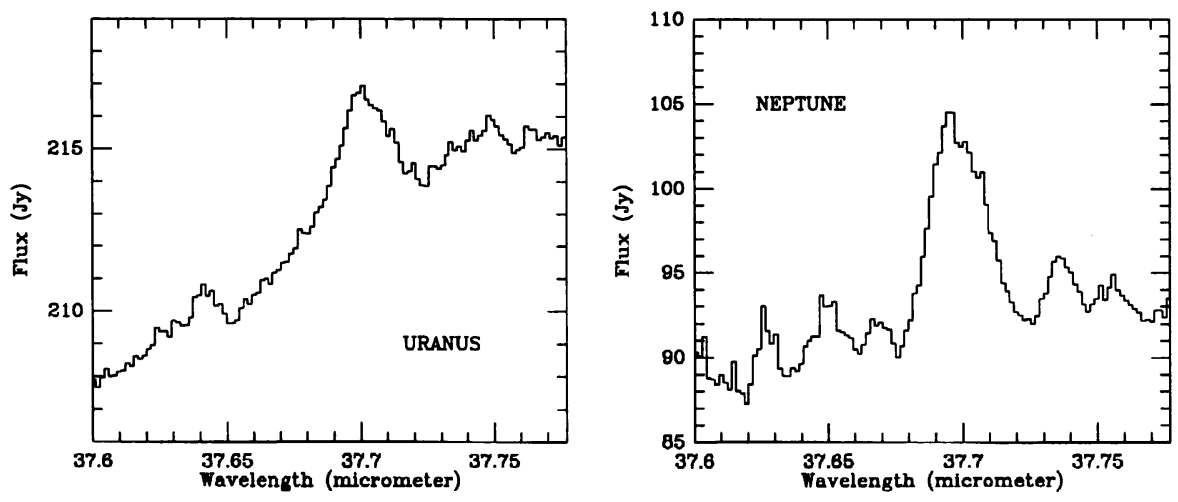

Figure 2. The first detection of HD rotational lines in Uranus and Neptune, from ISO/SWS observations of the R(2) transition. Inferred $\mathrm{D} / \mathrm{H}$ ratios are $(4-9) \times 10^{-5}$ for Uranus and $(5-9) \times 10^{-5}$ for Neptune. Adapted from Feuchtgruber et al. (1999a).

and Giant Planets (Bockelée-Morvan et al. 1998), and more generally of the structure of the protosolar nebula (Drouart et al. 1999).

New isotopic ratios in Jupiter are available for helium, carbon and nitrogen. The ${ }^{13} \mathrm{C} /{ }^{12} \mathrm{C}$ and ${ }^{3} \mathrm{He} /{ }^{4} \mathrm{He}$ ratios, determined by Galileo, have protosolar values. A very surprising result is that the ${ }^{14} \mathrm{~N} /{ }^{15} \mathrm{~N}$ ratio in Jupiter, as determined by ISO from inspection of $\mathrm{NH}_{3}$ lines at $10 \mu \mathrm{m}$, is equal to $525_{-170}^{+475}$ (Fouchet et al. 1999). This is essentially a factor of two larger than the terrestrial value (272) and 2.5 times larger than in the Solar atmosphere $\left({ }^{14} \mathrm{~N} /{ }^{15} \mathrm{~N}=200 \pm 55\right.$; Kallenbach et al. 1998). Fouchet et al. (1999) examined several atmospheric mechanisms that could plausibly modify the ${ }^{14} \mathrm{~N} /{ }^{15} \mathrm{~N}$ ratio (condensation and photolysis) but did not find a convincing scenario, so that the observed value may indeed reflect the bulk jovian ${ }^{14} \mathrm{~N} /{ }^{15} \mathrm{~N}$ ratio. This is however particularly difficult to interpret, both in the framework of a cometary origin for the planetesimals having formed Jupiter (the ${ }^{14} \mathrm{~N} /{ }^{15} \mathrm{~N}$ has a quasi-telluric value in comets, e.g. $323 \pm 46$ in Hale-Bopp; Jewitt et al. 1997), and in the framework of an origin from the protosolar gas nebula (in this case, the $\mathrm{N} / \mathrm{H}$ ratio should not be oversolar).

\section{Clouds and Deep Atmosphere Meteorology}

\subsection{Pre-Galileo expectations}

Galileo, and to a lesser extent ISO, have provided us with a wealth of new data on the meteorology of the deep atmosphere of the Giant Planets. In Giant Planets, nitrogen, oxygen and sulfur incorporated during the planetary formation are mainly in the form of tropospheric $\mathrm{NH}_{3}, \mathrm{H}_{2} \mathrm{O}$, and $\mathrm{H}_{2} \mathrm{~S}$, respectively. In the case of water, a stratospheric component is also present, as discussed below. Note that $\mathrm{H}_{2} \mathrm{~S}$ had been searched for extensively in Giant Planets, but could 
not be detected unambiguously prior to Galileo. The key point is that these three species are gaseous at deep and warm levels but condensible at the lower temperatures of the upper tropospheres. Specifically, simple models based on the thermodynamical equilibrium formation of clouds (the so-called Equilibrium Cloud Condensation Models (ECCM)) predict the existence of well-marked levels where these gases condense out and form clouds. In the case of Jupiter, for solar (or moderately oversolar) abundances, condensation is expected to occur near 5 bar $(275 \mathrm{~K})$ for $\mathrm{H}_{2} \mathrm{O}, 2$ bar $(210 \mathrm{~K})$ for $\mathrm{H}_{2} \mathrm{~S}$ which will precipitate in the form of ammonium sulfide ice, and 0.7 bar $(150 \mathrm{~K})$ for $\mathrm{NH}_{3}$. Similar layers are expected to form at similar temperatures (therefore somewhat deeper pressure levels) in Saturn and the other Giant Planets. In the absence of any physical phenomena besides condensation, the vertical profile of each of the condensible species is expected to be uniform below the base of the associated cloud, and to follow the vapor pressure equilibrium above. Based on terrestrial analogy, a moderate undersaturation can occur above the cloud base and is actually observed for ammonia in the cloudy regions of Jupiter (e.g. Lara et al. 1998). ECCMs, which do not include any microphysical processes such as precipitation, are unable to predict realistic cloud densities. Yet, they are considered to adequately describe the cloud positions in the conditions of adiabatic ascent of the air with condensation, and comparison with actual data is therefore enlightening.

\subsection{Galileo Probe results}

The Galileo Probe measured Jupiter's atmospheric composition, cloud and wind structure down to the 21 bar level. The most important point is that the measurements were performed in a hot spot. In visible light, or at near-infrared wavelengths (outside the strong gaseous absorptions), Jupiter exhibits moderate, factor-of-several, spatial contrasts in local surface brightness. The brightest regions correspond to maximum backscattering by clouds. At wavelengths near $5 \mu \mathrm{m}(4.6-5.2 \mu \mathrm{m})$, spatial contrasts appear much stronger, up to a factor of 100, with a global inversion of colors: regions that are bright in the visible are generally dark at $5 \mu \mathrm{m}$, and vice-versa. This comes from the fact that the 5 $\mu \mathrm{m}$ radiation from Jupiter is thermal; it is emitted deep in the 2-10 bar tropospheric range and appears particularly strong in places where it is not filtered by clouds. These regions of high $5 \mu \mathrm{m}$ flux, often concentrated in the North Equatorial Belt, are called hot spots (this denomination is somewhat improper since the flux contrasts are not due to atmospheric temperature variations). They correspond to cloudless regions, and although they can be limited in extent to less than $10 \%$ of the jovian disk, they dominate the overall $5 \mu \mathrm{m}$ radiation from Jupiter. The importance of $5 \mu \mathrm{m}$ spectroscopy has been recognized more than 20 years ago (see e.g. Kunde et al. 1982). The fact that radiation originates from deep levels is favorable for the study of minor species below the clouds and has led to the first detection of several species in Jupiter $\left(\mathrm{CO}, \mathrm{H}_{2} \mathrm{O}, \mathrm{GeH}_{4}, \mathrm{AsH}_{3}\right)$.

Falling in one of these hot spots (at $6.5 \mathrm{~N}$ planetocentric longitude and $1 \mathrm{~W}$ System III longitude), the Galileo Probe detected indeed a very tenuous cloud structure. The clouds layers however, were diffuse rather than well-marked, and not exactly at the expected altitudes. Specifically, the Probe nephelometer sensed a tenuous cloud from 1.0-1.34 bar and possibly a thin layer at 1.6 bar (Ragent et al. 1998). Additional structures were seen below this level as well as 
at $0.46-0.55$ bar. This latter structure is consistent with the tentative detection of a thin cloud at 0.5 bar by the Net Flux Radiometer (Sromovsky et al. 1998). Structures near 0.5 and 1.0-1.6 bar can be tentatively identified as $\mathrm{NH}_{3}$ and $\mathrm{NH}_{4} \mathrm{SH}$, although the nephelometer could not measure the cloud composition. The expected deep water cloud near 5 bar was clearly not present.

Probably the most surprising result of the Galileo Probe observations was in the vertical distribution of the three condensible species, which were found to increase with depth independently of the cloud levels. More precisely, the abundance of $\mathrm{NH}_{3}, \mathrm{H}_{2} \mathrm{~S}$ and $\mathrm{H}_{2} \mathrm{O}$ increased below their expected or actual saturation levels, i.e. in atmospheric regions where the ECCM predicted them to be vertically constant. At the expected cloud levels, all three species were found to be undersaturated by 2-4 orders of magnitude. The abundances of $\mathrm{NH}_{3}$ and $\mathrm{H}_{2} \mathrm{~S}$ increased downward until a "recovery" level was reached, below which they remained constant at what presumably corresponds to the true bulk abundance of $\mathrm{N}$ and $\mathrm{S}$ (these abundances are those on which the $\mathrm{N} / \mathrm{H}$ and $\mathrm{S} / \mathrm{H}$ elemental ratios of Table 1 are based). Note that the recovery level was different for the two species, about 8 bar for $\mathrm{NH}_{3}$ and 14 bar for $\mathrm{H}_{2} \mathrm{~S}$. The increase of the ammonia abundance is confirmed by the attenuation of the radio signal from the Probe (Folkner et al. 1998). The water mixing ratio increased continuously from less than $1 \mathrm{ppm}$ at the 4 bar level to about $600 \mathrm{ppm}$ at 19 bar (i.e. $\mathrm{O} / \mathrm{H}=0.35$ solar), where the last measurement occurred. Probably the recovery level was not yet reached, so that the bulk abundance of oxygen in Jupiter was not sensed.

\subsection{Galileo Orbiter results}

Information from the Galileo Orbiter came from the imaging system (SSI) and from the Near Infrared Mapping Spectrometer. The imaging system identified a nearly ubiquitous cloud (with important variations in the optical thickness) with base near $0.75 \mathrm{~km}$ (Banfield et al. 1998), attributed to ammonia condensation. This layer generally prevented to sense deeper levels, although a deeper cloud at $p \geq 4$ bar (presumably water ice) was seen in one region northwest of the Great Red Spot. The infrared spectrometer mapped the $5 \mu \mathrm{m}$ spectrum over various regions of Jupiter, mostly hot spots, and found that the abundance of water over hot spots varies by huge factors (up to 100) but remains always well below the saturation, at typically $0.07-7 \%$ humidity (Roos-Serote et al. 1998). A complex distribution of water over hot spots was found, with the center of the hot spot sometimes surrounded by a wetter collar. The $5 \mu \mathrm{m}$ spectrum of hot spots also excludes the presence of a deep water cloud in these regions, consistent with the Probe results.

\subsection{ISO results}

The ISO results nicely complement those from Galileo. First, the long-sought spectral signature of ammonia ice was finally found in Jupiter. ISO-SWS spectra show (Fig. 3) that rather surprisingly, Saturn is brighter than Jupiter at 2.9-3.1 $\mu \mathrm{m}$. This appears to be due to absorption and scattering in the strong $\mathrm{NH}_{3}$ band at 2.95 micron on Jupiter (Encrenaz et al. 1996). A two-cloud model of Jupiter, with $10 \mu \mathrm{m} \mathrm{NH}$ particles in the upper cloud, provides a good match to the spectrum (Brooke et al. 1998). 


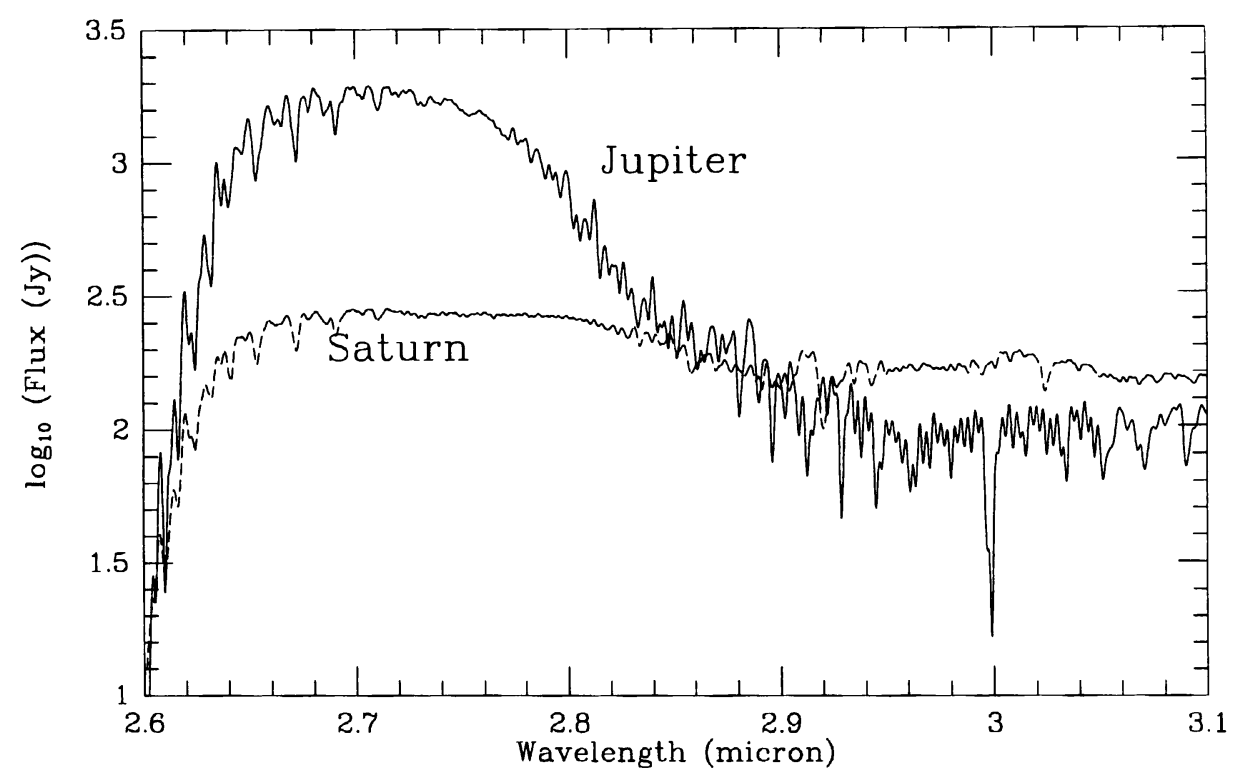

Figure 3. Comparison between the Jupiter and Saturn spectra, observed by ISO-SWS in the $3 \mu \mathrm{m}$ region. At 2.9-3.1 $\mu \mathrm{m}$, Saturn emits more photons than Jupiter, because ammonia ice strongly absorbs on Jupiter. Note the flux scale in log.

Second, a fine analysis of the ISO/SWS $5 \mu \mathrm{m}$ spectrum of Jupiter spectroscopically indicates that the $\mathrm{NH}_{3}$ mixing ratio increases with increasing depth (Fouchet et al. 1999). Since the spectrum is dominated by hot spot radiation, this strongly confirms the Galileo Probe results.

Finally, ISO allowed the first detection of deep water in Saturn, again from $5 \mu \mathrm{m}$ spectroscopy. As on Jupiter, the spectrum sounds Saturn's troposphere in the $2-5$ pressure range, where water is limited by condensation. Similar to Jupiter, the inferred amount of water is only $\sim 1.5 \%$ of the value allowed by the vapor pressure equilibrium. Although on Saturn hot spots are not clearly identified from $5 \mu \mathrm{m}$ imaging, this suggests that similar processes operate as on Jupiter to produce small, cloudless, volatiles-depleted areas dominating the 5 $\mu \mathrm{m}$ emission from the planet.

\subsection{Summary}

Interpreting all this new information on the deep atmosphere of Jupiter and to a lesser extent Saturn is challenging. A general conclusion is that hot spots are regions of descending motions. Outside hot spots, convective motions carry volatiles upwards up to a level where they condense. In hot spots, downward motions may then transport this dessicated air. Models based on this "downdraft" scenario (see e.g. Atreya et al. 1999; Baker \& Schubert 1998; Showman \& Ingersoll 1998) qualitatively explain the low abundance of volatiles in hot spots. However, they fail to quantitatively account for the different vertical profiles 
and the staggered recovery levels for the various volatiles. In addition, dry air is less dense on Jupiter than moist air, thus transporting downward dessicated air requires large, organized vertical winds $\left(10-15 \mathrm{~m} \mathrm{~s}^{-1}\right)$ over several scale heights, which are not supported by the Galileo Probe measurements. Until these inconsistencies are solved, it will be extremely difficult to estimate the bulk abundance of oxygen in Jupiter from the Galileo Probe data.

\section{Stratospheric Chemistry and Dynamics}

\subsection{Hydrocarbons in Giant Planet stratospheres}

Our knowledge of hydrocarbons in Giant Planet stratospheres has been considerably enhanced by the ISO-SWS observations. Several new hydrocarbons have been detected, particularly on Saturn $\left(\mathrm{CH}_{3} \mathrm{CCH}, \mathrm{C}_{4} \mathrm{H}_{2}, \mathrm{C}_{6} \mathrm{H}_{6}\right)$ (de Graauw et al. 1997; Bézard et al. 1999a; Fig. 1). The first detection of a radical, $\mathrm{CH}_{3}$, in a planetary atmosphere (in Saturn and Neptune; Bézard et al. 1998, 1999b) is also noteworthy. Information is available on the vertical profile of several species $\left(\mathrm{CH}_{4}, \mathrm{C}_{2} \mathrm{H}_{2}\right.$ and $\left.\mathrm{C}_{2} \mathrm{H}_{6}\right)$. For $\mathrm{C}_{2} \mathrm{H}_{2}$ and $\mathrm{C}_{2} \mathrm{H}_{6}$, information is obtained, classically, from the comparison of weak/strong line intensities in optically thick bands. For methane at Jupiter and Saturn, the information comes mainly from the first detection of non-LTE fluorescence emission at $3.3 \mu \mathrm{m}$ (Drossart et al. 1999), an exceptional "comet-like" situation in a planetary atmosphere.

Detecting new molecules and deriving vertical information is more than adding new poststamps in a collection. Hydrocarbons in Giant Planet stratospheres are produced from the photochemistry of methane and their distribution results not only from chemical schemes but also from the strength of vertical transport. Vertical transport results from a combination of molecular and turbulent diffusion, the latter being parameterized by a so-called eddy (i.e. turbulent) diffusion coefficient. Different minor species are sensitive to the value of the eddy diffusion in different pressure ranges, allowing to reconstruct the strength of the vertical transport as a function of altitude. This in turn may provide information on the source of turbulence in planetary atmospheres. In addition, current models (see Fig. 4 for the chemical scheme of the most recent, postISO photochemical of Saturn; Moses et al. 1999a) are now elaborate enough so that critical pathways controlling minor species abundances can be identified. For a number of them, better measurements of kinetic rates from the laboratory are desirable. Examples are some radical-radical reactions: $\mathrm{CH}_{3}+\mathrm{H} \longrightarrow$ $\mathrm{CH}_{4} ; \mathrm{CH}_{3}+\mathrm{CH}_{3}+\mathrm{M} \longrightarrow \mathrm{C}_{2} \mathrm{H}_{6} ; \mathrm{H}+\mathrm{C}_{2} \mathrm{H}_{3} \longrightarrow \mathrm{C}_{2} \mathrm{H}_{2}+\mathrm{H}_{2} ; \mathrm{H}+\mathrm{C}_{2} \mathrm{H}_{3}+\mathrm{M} \longrightarrow$ $\mathrm{C}_{2} \mathrm{H}_{4}+\mathrm{M}$; and $\mathrm{H}+\mathrm{C}_{2} \mathrm{H}_{4}+\mathrm{M} \longrightarrow \mathrm{C}_{2} \mathrm{H}_{5}+\mathrm{M}$ (see Bézard et al. 1998; Moses et al. 1999a).

\section{External Supply of Oxygen}

While most of the oxygen of the Giant Planets is trapped as tropospheric water (and presumably silicates at deeper levels), there is also an external oxygen component. Indeed, water ice or other oxygen-bearing material $\left(\mathrm{CO}, \mathrm{CO}_{2}\right.$ ices, clathrates, silicates) coming from the interplanetary medium (interplanetary dust, asteroids in all ranges of size, comets) or from planetary environments 


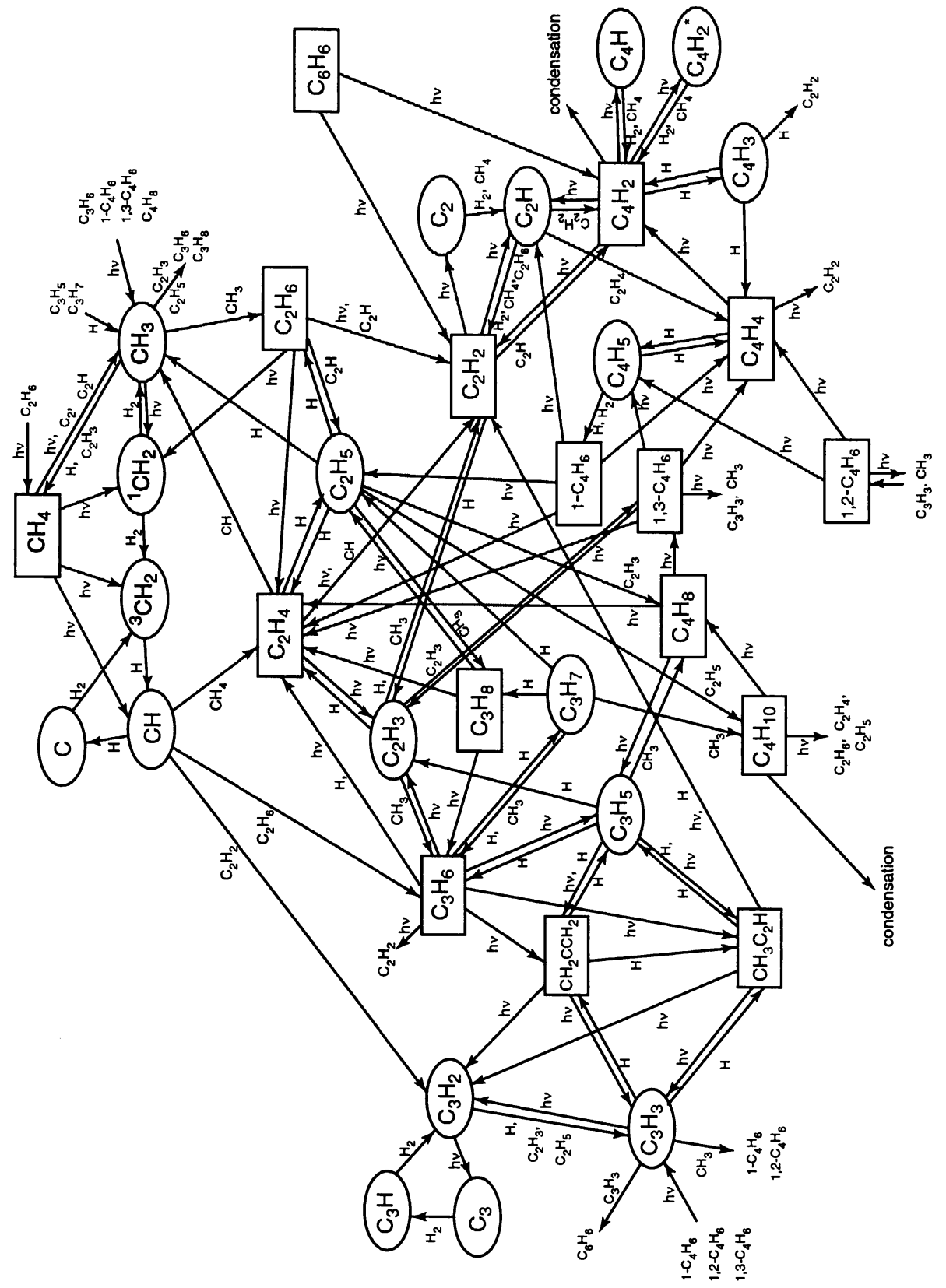

Figure 4. A diagram illustrating the hydrocarbon chemistry in Saturn's atmosphere (from Moses et al. 1999). 

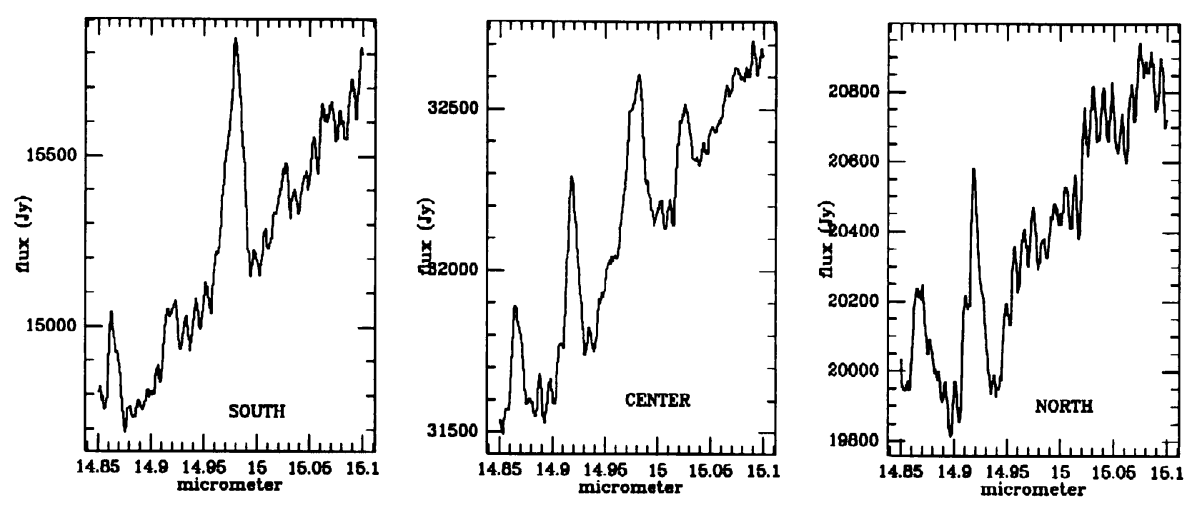

Figure 5. Three ISO-SWS spectra of Jupiter in the $15 \mu \mathrm{m}$ region, showing variations of the $\mathrm{CO}_{2}$ emission intensity with latitude.

(rings, satellites) may infall onto the planet and be vaporized. Similar to the internal water that is carried upward by convection and condense in the upper troposphere, the external water can be transported downward and condense in the lower stratosphere.

One of the most important results of ISO in the Solar System has been the first detection by SWS of this external water in all four Giant Planet stratospheres and Titan, along with $\mathrm{CO}_{2}$ on Jupiter, Saturn and Neptune. Typical water and $\mathrm{CO}_{2}$ mixing ratios above the condensation levels (which occur in the millibar-microbar range) are $10^{-9}$. The order of magnitude of the input flux, which is the physically important parameter, can be estimated from a simple transport model. Resulting fluxes, in mol $\mathrm{cm}^{-2} \mathrm{~s}^{-1}$, are $(0.6-1.8) \times 10^{6},(0.3-$ $5) \times 10^{6},(0.8-2.8) \times 10^{6},(0.06-0.16) \times 10^{6}$ and $(0.12-15) \times 10^{6}$ for Jupiter, Saturn, Titan, Uranus and Neptune respectively (Feuchtgruber et al. 1997, 1999b; Coustenis et al. 1998). They thus appear similar on different planets, particularly on Jupiter, Saturn and Titan. The admittedly large uncertainty in some cases is mostly a consequence of the uncertainty on the vertical transport loss, which acts as a vacuum cleaner for the atmospheric water. This also illustrates the need to understand upper atmosphere dynamics.

In the case of Saturn, an improved modelling is now available (Moses et al. 1999b), making use of the eddy diffusion coefficient profile deduced from the hydrocarbons, as discussed above, and including the full chemistry coupling oxygen and hydrocarbon compounds. Its major results are as follows: (i) to explain the ISO observations of $\mathrm{H}_{2} \mathrm{O}$ and $\mathrm{CO}_{2}$ at Saturn, the oxygen cannot enter the atmosphere only in the form of water; an additional flux of oxygen in the form of a $\mathrm{C}-\mathrm{O}$ bearing molecule $\left(\mathrm{CO}, \mathrm{CO}_{2}, \mathrm{CH}_{3} \mathrm{OH}\right)$ is required; (ii) although the relative proportion of $\mathrm{H}_{2} \mathrm{O}$ and this additional species are difficult to assess precisely (10-90\% for each of them), the total oxygen flux can now be constrained to within a factor of 2 to be $(4 \pm 2) \times 10^{6} \mathrm{O}$ atoms $\mathrm{cm}^{-2} \mathrm{~s}^{-1}$, i.e. $(1 \pm 0.5) \times 10^{-16}$ $\mathrm{g} \mathrm{cm}^{-2} \mathrm{~s}^{-1}$; (iii) if allowance is made for the non-ice, organic-rich, component of comets, then an incoming material consistent with the composition of comets 
is able to account for observations; (iv) a global, planet-wide influx of oxygen gives a better match to the data than a localized flux that would result, notably, from precipitation of ring material at specific latitudes.

From these results, one can attempt to establish the origin of the external water. The following discussion, based on Moses et al. (1999), applies to Saturn but can largely be extended to other Giant Planets. Possible sources include:

- "Large" comets: large (Shoemaker-Levy 9 type) cometary impacts are estimated to occur once every 300-800 years at Saturn. From the SL9 experience, about $3 \%$ of the total cometary mass ends up as atmospheric water, while $30 \%$ of the mass (i.e. most of the cometary water) is converted to $\mathrm{CO}$ by shock chemistry at impact. These numbers result in equivalent fluxes of $(2-5) \times 10^{-17} \mathrm{~g} \mathrm{~cm}^{-2} \mathrm{~s}^{-1}$ of $\mathrm{CO}$ and ten times less $\mathrm{H}_{2} \mathrm{O}$. This is significant; however the lifetime of $\mathrm{H}_{2} \mathrm{O}$ in Saturn's atmosphere is only 15 years, so it is unlikely that Saturn's current $\mathrm{H}_{2} \mathrm{O}$ results from a large cometary impact. In contrast, this source appears to be important for Jupiter's $\mathrm{CO}_{2}$, as ISO-SWS observations show a strong latitudinal asymmetry in the $\mathrm{CO}_{2} 15 \mu \mathrm{m}$ emission, consistent with the distribution of minor species 3-5 years after the SL9 impacts (Fig. 5).

- "Small" comets: there exists a whole distribution of population of comets vs. size. The cumulative number of objects larger than $D(\mathrm{~km})$ and hitting Saturn can be estimated to $0.003 \mathrm{D}^{-2}$ per year. For $\mathrm{D}=0.22 \mathrm{~km}$, the time interval between impacts is 15 years, meeting the lifetime requirement. However, the mass flux for comets smaller than $0.22 \mathrm{~km}$ is 10 times too small.

- Interplanetary dust: at the lower end of the mass range, the flux of interplanetary dust particles (IDP) is 30,000 tons $\mathrm{yr}^{-1}$ at the Earth. Extrapolating to Saturn's distance, and accounting for the gravitational focussing by Saturn leads to a flux into Saturn of $(1-20) \times 10^{-16} \mathrm{~g} \mathrm{~cm}^{-2} \mathrm{~s}^{-1}$. Although the uncertainty is large, due mostly to the uncertain particle velocity at Saturn's distance, this is probably a viable source. In contrast, the flux of interstellar dust, is about 4 orders of magnitude too small, at least for small $\left(3 \times 10^{-13} \mathrm{~g}\right)$ particles (large particles have not been measured).

- Ring source: planetary rings can be eroded by IDP impact, producing vapor and dust. Both components are then partly ionized and transported to the planet either through capture along magnetic lines or from Poynting-Robertson drag. This source is therefore proportional to the direct IDP source, and the ratio between the two depends essentially on the erosion efficiency (i.e. the ratio of excavated mass to incident mass) and the transport efficiency (i.e. the fraction of the mass leaving the ring that will eventually reach the planet). Based on estimates from Morfill et al. (1983), the ring vapor source appears negligible; in contrast, the ring dust source may be important and even dominant at some specific latitudes. This source may have been detected in HST observations (Prangé et al. 1998).

Overall, given the above considerations, the interplanetary dust appears to be the most likely to account for Saturn's external oxygen. Adapting the full- 
blown photochemical model to the other Giant Planets will allow one to refine the fluxes and interpret them in the context of this hypothesis. Ultimately, the goal will be to determine the source of this interplanetary dust at 5-30 AU. Possible origins include (i) cometary activity at large heliocentric distances; and (ii) collisions between Kuiper Disk objects. Thus, the problem of external supply of oxygen to the Giant Planets may have far-reaching implications.

\section{References}

Atreya, S.K., et al. 1999, Planet. Space Sci., 47, 1243

Baker, R.D. \& Schubert G. 1998, Icarus, 136, 340

Banfield, D., et al. 1998, Icarus, 135, 230

Bézard, B., Feuchtgruber, H., Moses, J.I., \& Encrenaz T. 1998, A\&A, 334, L41

Bézard, B., Feuchtgruber, H., \& Encrenaz T. 1999a, in The Universe as Seen by ISO, ESA-SP, 427, 153

Bézard, B., et al. 1999b, ApJ, 515, 868

Bockelée-Morvan D., et al. 1998, Icarus, 133, 147

Brooke, T.Y., Knacke, R.F., Encrenaz, Th., et al. 1998, Icarus, 136, 1

Coustenis, A., Salama, A., Lellouch, E., et al. 1998, A\&A, 336, L85

Drossart, P., et al 1999, in The Universe as Seen by ISO, ESA-SP, 427, 169

Drouart, A., Dubrulle, B., Gautier, D., \& Robert, F. 1999, Icarus, 140, 129

Encrenaz, T., et al. 1996, A\&A, 315, L397

Feuchtgruber, H., et al. 1997, Nature, 389, 159

Feuchtgruber, H., et al. 1999a, A\&A, 341, L17

Feuchtgruber, H., et al. 1999b, in The Universe as Seen by ISO, ESA-SP, 427, 133

Fouchet, T., et al. 1999, Icarus, submitted

Folkner, W.M., Woo, R., \& Nandi, S. 1998, J. Geophys. Res., 103, 22847

Gautier, D. 1998, In Nantes Symposium (11-15 May 1998)

de Graauw, T., Feuchtgruber, H., Bézard, B., et al. 1997, A\&A, 321, L13

Jewitt, D.C., Matthews, H.E., Owen, T., \& Meier, R. 1997, Science, 278, 90

Kallenbach, R., et al. 1998, ApJ, 507, L185

Kunde, V., et al. 1982, ApJ, 263, 443

Lara, L-M., et al. 1998, Icarus, 131, 317

Lellouch, E. 1999, in The Universe as Seen by ISO, ESA-SP, 427, 125

Mahaffy, P.R., et al. 1998, Space Sci. Rev, 84, 251

Morfill, G.E., Fechtig, H., Gruen, E., \& Goertz, C.K. 1983, Icarus, 55, 439

Moses, J.I., et al. 1999a, Icarus, submitted

Moses, J.I., et al. 1999b, Icarus, submitted

Niemann, H.H., 1996, Science, 272, 846

Niemann, H. H., et al. 1998, J. Geophys. Res., 103, 22831

Prangé, R., et al. 1998, in Nantes Symposium (11-15 May 1998)

Ragent, B., et al. 1998, J. Geophys. Res., 103, 22891

Roos-Serote, M., et al., 1998, J. Geophys. Res., 103, 23023

Showman, A.P. \& Ingersoll, A.P. 1998, Icarus, 132, 205

Sromovsky, L.A., et al. 1998, J. Geophys. Res., 103, 22929 


\section{Discussion}

W. M. Irvine: Can you comment on the ${ }^{14} \mathrm{~N} /{ }^{15} \mathrm{~N}$ ratio for Jupiter, the Sun, and the local ISM?

E. B. Lellouch: A difficult question. ISO finds a ${ }^{14} \mathrm{~N} /{ }^{15} \mathrm{~N}$ ratio in Jupiter of about 500, while the solar value, as mentioned by Owen, is about 200 . It is very surprising to get different numbers. In fact, the Jupiter ${ }^{14} \mathrm{~N} /{ }^{15} \mathrm{~N}$ appears to be similar to the value measured in the local ISM for HCN but this may not be relevant.

F. van der Tak: The spectroscopic detection of $\mathrm{NH}_{3}$ ice on Jupiter with the ISO-SWS is a very nice result. Would the same be possible on Saturn?

E. B. Lellouch: Yes, in principle it is possible. However the $3 \mu \mathrm{m}$ region spectrum of Saturn does not show the $\mathrm{NH}_{3}$ ice signature as on Jupiter. Although there must be $\mathrm{NH}_{3}$ clouds on Saturn also, they are expected to occur somewhat deeper than on Jupiter (about 1.5 bar for Saturn vs. 0.7 bar for Jupiter). So it means that the $3 \mu \mathrm{m}$ radiation on Saturn does not penetrate that deep. Another difference is the large phosphine absorption in Saturn in this region, which may contribute to hiding the clouds.

M. Guélin: The ISO $\mathrm{CO}_{2}$ spectra obtained toward the Jupiter $\mathrm{N}$ and S poles are impressive. Did you have the opportunity to check that they are really linked to SL9 by taking spectra toward the rising and setting points of the equator?

E. B. Lellouch: We have not taken spectra on the equatorial limbs, but we have a sequence of spectra taken at the disk center and different longitudes on Jupiter (simply by letting Jupiter rotate). We see no variation with longitude in the $\mathrm{CO}_{2}$ emission. This is not surprising since long term monitoring of the $\mathrm{CO}$ and CS emission at millimeter wavelengths indicates their longitudinal structure has disappeared in the impact band at $45^{\circ}$ South latitude. 